\title{
Efficacy and Safety of Vedolizumab in Ulcerative Colitis and Crohn's Disease Patients Stratified by Age
}

\author{
Vijay Yajnik · Nabeel Khan · Marla Dubinsky · Jeffrey Axler • \\ Alexandra James · Brihad Abhyankar · Karen Lasch
}

Received: September 16, 2016/Published online: January 9, 2017

(C) The Author(s) 2017. This article is published with open access at Springerlink.com

\begin{abstract}
Introduction: The efficacy and safety of vedolizumab, a gut-selective $\alpha_{4} \beta_{7}$ integrin antibody, were demonstrated in the GEMINI 1 and GEMINI 2 clinical trials of adults aged $18-80$ years. We investigated the efficacy and safety of vedolizumab in patients stratified by age from the GEMINI trials.

Methods: Safety and efficacy, including clinical response, clinical remission, and corticosteroidfree remission, at week 6 and/or 52 were determined post hoc in patients aged $<35,35$ to $<55$, and $\geq 55$ years.
\end{abstract}

Enhanced content To view enhanced content for this article go to http://www.medengine.com/Redeem/ 1B47F0606B45CCEA.

Electronic supplementary material The online version of this article (doi:10.1007/s12325-016-0467-6) contains supplementary material, which is available to authorized users.

V. Yajnik $(\bowtie)$

Massachusetts General Hospital, Harvard Medical

School, Boston, MA, USA

e-mail:VYAJNIK@mgh.harvard.edu

N. Khan

Perelman School of Medicine, University of

Pennsylvania, Philadelphia, PA, USA

M. Dubinsky

Mount Sinai Hospital, New York, NY, USA
Results: At baseline, 353, 412, and 130 ulcerative colitis (UC) and 582, 443, and 90 Crohn's disease (CD) patients were aged $<35,35$ to $<55$, and $\geq 55$. Of these patients, 56 were aged $\geq 65$ years (UC: $33, \mathrm{CD}: 23$ ). Trends favoring vedolizumab over placebo were observed for most efficacy endpoints irrespective of patient age; some variability between subgroups was observed. Safety profiles of vedolizumab and placebo were similar in all age groups. Vedolizumab-treated patients aged $\geq 55$ had the lowest incidence of serious infections (0.9 per 100 person-years) and adverse events leading to hospitalization (14.8 per 100 person-years). There were no age-related differences in the incidence of adverse hematological events, malignancy, or death.

Conclusions: The safety and efficacy of vedolizumab in patients with $\mathrm{UC}$ or $\mathrm{CD}$ were similar for all age groups. The number of patients in the oldest age group in these analyses was small; thus further studies of

J. Axler

Toronto Digestive Disease Associates, University of

Toronto, Toronto, Canada

A. James - B. Abhyankar

Takeda Development Centre Europe Ltd, London, UK

K. Lasch

Takeda Pharmaceuticals U.S.A. Inc, Deerfield, IL, USA 
vedolizumab in larger cohorts of elderly patients are warranted.

Funding: Millennium Pharmaceuticals, Inc. (d/b/a Takeda Pharmaceuticals International Co.).

Keywords: Crohn's disease; Ulcerative colitis; Vedolizumab

\section{INTRODUCTION}

Inflammatory bowel disease (IBD) is a disorder of the gastrointestinal tract that includes ulcerative colitis (UC) and Crohn's disease (CD) $[1,2]$. The prevalence of IBD in elderly patients is high, with $10-30 \%$ of the global IBD population over the age of 60 [3]. The epidemiology, clinical characteristics, and natural history of IBD are heterogeneous with respect to age of onset, and thus treatment should be individualized, including considerations for age [4]. However, safe and effective therapeutic options for aging IBD patients are limited and treatment is challenging, confounded by age-related comorbidities, immunodeficiency, and overall elevated rates of polypharmacy $[5,6]$. Consequently, the incidence of treatmentrelated adverse events and overall rates of mortality and morbidity are higher in older IBD patients $[7,8]$.

The therapeutic management of IBD in aging populations is an active area of research, and numerous studies have demonstrated marked deficiencies and risks associated with the use of several IBD treatments [6, 8-14]. For example, anti-tumor necrosis factor (anti-TNF) therapies, corticosteroids, and thiopurines-as individual treatment regimens or in combination-are associated with higher risks of malignancy and opportunistic infections in elderly IBD patients than their younger counterparts $[8,9,11$, 15-17]. Increased infection rates with anti-TNF therapy in elderly IBD patients have, in turn, been proposed as an underlying reason for high rates of IBD treatment discontinuation [10] and increased risk of mortality related to hospitalizations [8]. Further, long-term, high-frequency, or elevated-dose use of corticosteroids-common in the elderly population-may exacerbate several cardiometabolic and cognitive conditions $[14,18]$. Oral corticosteroid therapy in elderly patients is also linked to diabetes mellitus $[6,19]$, which confers a nearly twofold increased risk of infections such as sepsis, pneumonia, and urinary tract infections in patients treated with immunosuppressants [13]. Rates of non-melanoma skin cancer and risk of lymphoma associated with thiopurine use were found to increase with increasing age [17] or with successive years of therapy [16], making long-term use undesirable.

Vedolizumab is a novel humanized immunoglobulin $G_{1}$ monoclonal antibody to $\alpha_{4} \beta_{7}$ integrin that selectively inhibits inflammation in the gastrointestinal tract without inhibiting systemic immune responses [20]. This gut-selective mechanism of action may be particularly relevant to aging populations because of their elevated risk of systemic infections and malignancy [16, 21]. The safety and efficacy of vedolizumab for moderately to severely active UC and CD were demonstrated in the phase 3 GEMINI 1 and GEMINI 2 studies, respectively [22, 23]. However, aside from an analysis of the role of age as a risk factor for infection for vedolizumab-treated patients with $\mathrm{UC}$ or $\mathrm{CD}$ [24], limited data exist on the safety and efficacy of vedolizumab in older populations in part because few patients over the age of 65 years enrolled in the GEMINI 1 and GEMINI 2 studies. In this paper we use available study data to report post hoc analyses of the safety and efficacy of vedolizumab in patients grouped by age. In an effort to include meaningful numbers of patients in each age stratum, we divided the population as follows: aged $<35,35$ to $<55$, and $\geq 55$ years. Recognizing that the elderly population typically refers to those aged $\geq 65$ years, we provide safety and efficacy data for GEMINI patients in this age group as well.

\section{METHODS}

These results are based on post hoc subgroup analyses of data from the GEMINI 1 and GEMINI 2 studies (ClinicalTrials.gov 
identifiers: NCT00783718 and NCT00783692, respectively) which enrolled patients aged 18-80 years with moderately to severely active $\mathrm{UC}$ or CD, respectively $[22,23]$. We retrospectively investigated the safety and efficacy of vedolizumab in patients stratified into the following age groups: $<35,35$ to $<55$, and $\geq 55$ years. These data are also reported for the age groups $<65$ and $\geq 65$.

\section{Study Designs}

The GEMINI 1 and GEMINI 2 study designs have been previously published [22, 23]. Patients received double-blind vedolizumab or placebo [cohort 1; induction intent-to-treat (ITT) population] or open-label vedolizumab (cohort 2) during the induction phase (Supplementary Fig. S1). Patients from either cohort who demonstrated clinical response to vedolizumab at week 6 received vedolizumab every 8 weeks or every 4 weeks or placebo up to week 52 beginning at week 6 (maintenance ITT population) (Supplementary Fig. S1). Patients who did not have clinical response at week 6 with vedolizumab induction therapy and patients who had received placebo could continue with vedolizumab every 4 weeks or placebo, respectively, for the remainder of the study (Supplementary Fig. S1). Patients receiving stable doses of corticosteroids at study baseline were permitted to continue treatment, but had to begin a defined steroid tapering regimen once response to vedolizumab was achieved. At centers outside the USA, patients were permitted to continue stable doses of immunosuppressants throughout the study. However, in the USA, patients were required to discontinue immunosuppressant use after the induction phase. Patients who completed or withdrew early from GEMINI 1 or GEMINI 2 during the maintenance phase were eligible to enroll in the open-label extension trial, GEMINI Long-term Safety (LTS; ClinicalTrials.gov identifier: NCT00790933). All patients received open-label vedolizumab every 4 weeks during GEMINI LTS.

\section{Efficacy Analyses}

The primary and secondary efficacy endpoints from the GEMINI 1 and GEMINI 2 studies were evaluated post hoc in patients according to their age at baseline and compared descriptively.

The primary endpoint for the GEMINI 1 induction phase was clinical response [reduction in the complete Mayo Clinic score of $\geq 3$ points and $\geq 30 \%$ from baseline (week 0 ) with an accompanying decrease in rectal bleeding subscore of $\geq 1$ point or absolute rectal bleeding subscore of $\leq 1$ point] at week 6. Secondary endpoints were clinical remission (complete Mayo Clinic score of $\leq 2$ and no subscore of $>1$ ) and mucosal healing (Mayo Clinic endoscopic subscore of 0 or 1 ) at week 6 . The primary endpoint for the GEMINI 1 maintenance phase was clinical remission at week 52 . Secondary endpoints at week 52 were durable clinical response (clinical response at both weeks 6 and 52), durable clinical remission (clinical remission at both weeks 6 and 52), mucosal healing, and corticosteroid-free remission (clinical remission without corticosteroid therapy in patients receiving corticosteroids at baseline) [23].

The primary endpoints for the GEMINI 2 induction phase were clinical remission (CD Activity Index (CDAI) score of $\leq 150$ points) and enhanced clinical response $(\geq 100$-point decrease in the CDAI score from baseline) at week 6 . The secondary endpoint at week 6 was the mean change in C-reactive protein (CRP) levels from baseline. The primary endpoint for the GEMINI 2 maintenance phase was clinical remission at week 52, and the secondary endpoints at week 52 were enhanced clinical response, corticosteroid-free remission (clinical remission without corticosteroid therapy in patients receiving corticosteroids at baseline), and durable clinical remission (clinical remission at $\geq 80 \%$ of study visits, including the final visit) [22].

For each binary endpoint, the absolute differences in percentages of patients meeting the endpoint with vedolizumab and placebo 
were calculated, along with 95\% confidence intervals (CIs). For the continuous efficacy endpoint (i.e., CRP levels), the mean change from baseline was calculated for each treatment group using the last observation carried forward method and reported with its standard deviation. For the week 52 efficacy endpoints, the maintenance ITT vedolizumab every 8 week and every 4 week groups were combined in each study to increase the patient population of each subgroup.

\section{Safety Analyses}

Safety data were collected from week 0 through week 52 for all patients who received vedolizumab at any time during the study and all patients who received placebo only, and data from both studies were pooled. Adverse events were classified according to the Medical Dictionary for Regulatory Activities (MedDRA), version 14.0 [25]. Patients were monitored for infusion-related reactions during and after the infusion and were also instructed to alert investigators of the development of such reactions, including rash, hives, and pruritus. Adverse events that were considered to be infusion-related by the investigator were recorded. In addition, malignancies, deaths, and treatment-emergent adverse events leading to hospitalizations were reported. Of note, all safety results were based on a post hoc subgroup analysis of data from the GEMINI 1 and GEMINI 2 studies, neither of which was powered for safety analyses. Therefore, descriptive statistics were used to compare incidences of adverse events between the different groups and no statistical conclusions were made. Adverse events were presented as the number of patients with the event and as exposure-adjusted incidence rates, defined as the number of patients experiencing the event per 100 person-years (PY) of exposure. Exposure was calculated separately for each adverse event as the date of onset minus the date of first dose plus 1. PY of exposure were calculated for the first occurrence of each adverse event, and truncated after a patient experienced the adverse event. However, the patient could continue to contribute PY for different adverse events. Thus, the total PY of exposure for each adverse event could differ.

\section{Ethical Considerations}

The study protocol and the informed consent documentation were reviewed and approved by the institutional review board(s) or independent ethics committee(s) at each investigational center. The study was conducted in compliance with the protocol, good clinical practice, and the applicable regulatory requirements (including International Conference on Harmonisation guidelines), and in accordance with ethical principles founded in the Declaration of Helsinki of 1964, as revised in 2013. Written informed consent for being included in the study was obtained from all patients at the time they entered the screening process.

\section{RESULTS}

\section{Patients}

The majority of patients enrolled in either GEMINI 1 or GEMINI 2 were $<55$ years of age (Fig. 1 and Supplementary Fig. S1). GEMINI 1 enrolled 895 patients with moderately to severely active UC, of whom 39\%, 46\%, and $15 \%$ were aged $<35,35$ to $<55$, and $\geq 55$ years, respectively (Fig. 1a). GEMINI 2 enrolled 1115 patients with moderately to severely active $\mathrm{CD}$, of whom $52 \%, 40 \%$, and $8 \%$ were $<35,35$ to $<55$, and $\geq 55$ years of age (Fig. 1b). In GEMINI 1 and GEMINI 2 studies, 33 (4\%) and 23 (2\%) patients, respectively, were aged $\geq 65$ years. There were no striking differences in the distribution of ages between the treatment groups in either study.

Differences between age groups were not evaluated statistically and are characterized descriptively in Tables 1 and 2. Overall, baseline characteristics of the induction safety populations-in particular concomitant medications-were similar across the age groups, except for disease duration (Tables 1 
(a)

UC Patients (GEMINI 1, N=895)

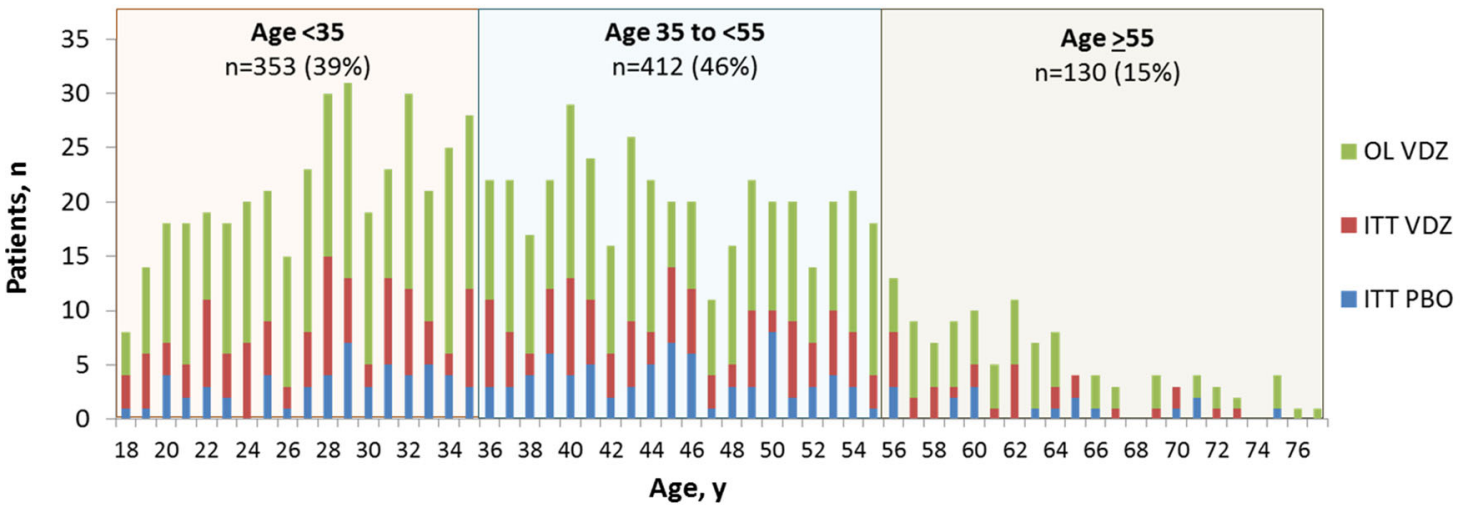

(b)

CD Patients

(GEMINI 2, N=1115)

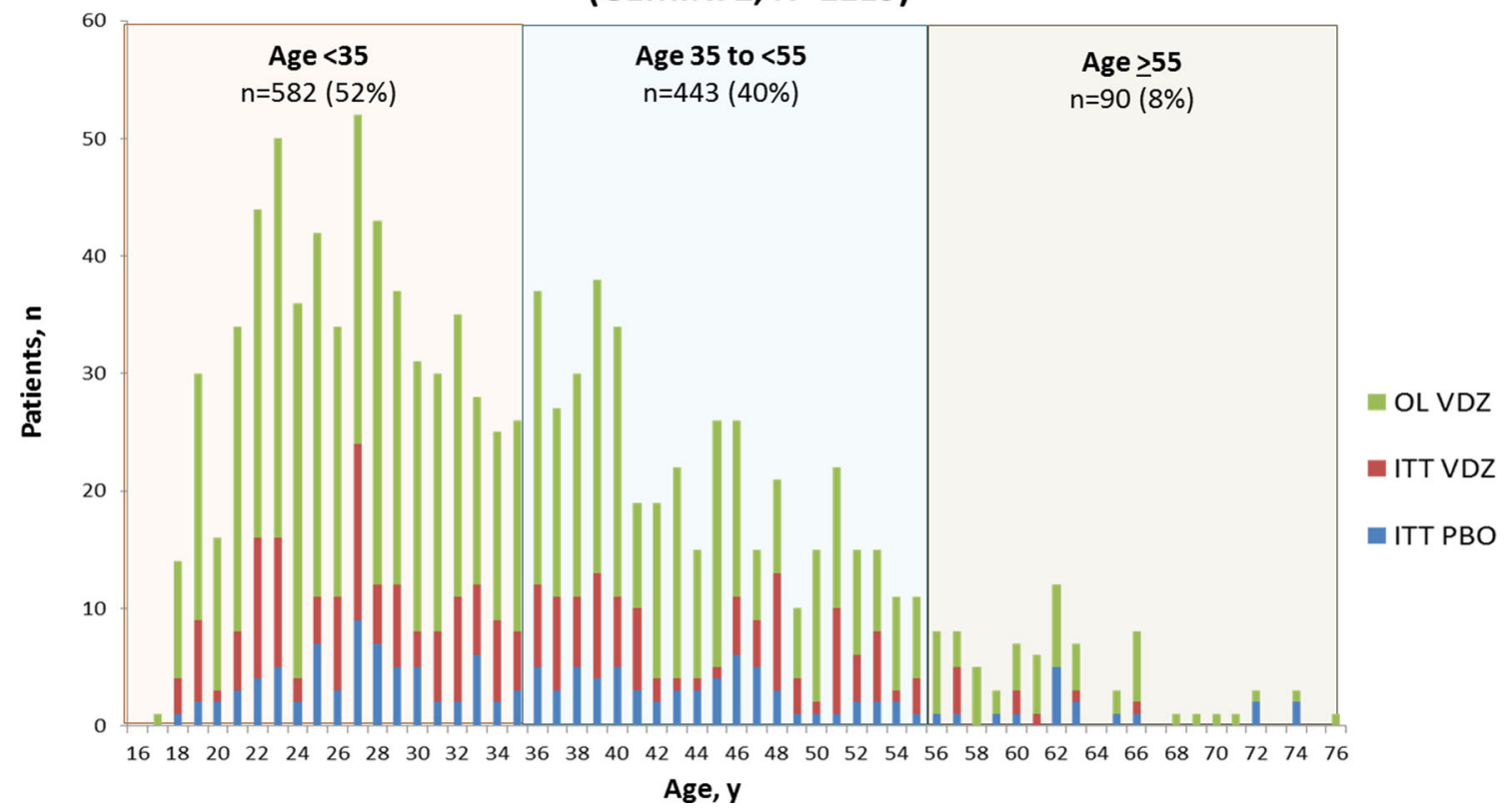

Fig. 1 Age of the patient population in GEMINI 1 and GEMINI 2. Number of patients at each age at baseline in a GEMINI 1 and b GEMINI 2 according to induction

and 2). In addition, disease location varied and disease activity scores were lower for CD patients $\geq 55$ years old (Table 2 ). The mean disease duration in UC patients aged $<35$ years was $<5$ years, whereas the mean in patients aged 35 to $<55$ years and those aged $\geq 55$ years was 8 and $\geq 9$ years, respectively (Table 1 ). Mayo Clinic scores were consistent across the age groups (Table 1). The mean duration of CD treatment received. $C D$ Crohn's disease, ITT intent-totreat, $O L$ open-label, $P B O$ placebo, $U C$ ulcerative colitis, $V D Z$ vedolizumab

ranged from $<7$ years in patients $<35$ years old to $>12$ years in patients $\geq 55$ years old (Table 2 ). Similarly, mean CDAI scores ranged from $\geq 325$ in CD patients $<35$ years old to $<310$ in those $\geq 55$ (Table 2 ). In addition, a greater percentage of CD patients aged $<35$ years had CD located in both the ileum and the colon than those aged $\geq 35$ years (Table 2 ). After re-randomization of vedolizumab responders at week 6 , baseline 
Table 1 UC induction population demographics and baseline disease characteristics

\begin{tabular}{|c|c|c|c|c|c|c|}
\hline \multirow[t]{2}{*}{ Characteristic } & \multicolumn{2}{|c|}{ Age $<35$ years } & \multicolumn{2}{|c|}{ Age 35 to $<55$ years } & \multicolumn{2}{|c|}{ Age $\geq 55$ years } \\
\hline & $\begin{array}{l}\text { PBO } \\
(n=53)\end{array}$ & $\begin{array}{l}\mathrm{VDZ}^{\mathrm{a}} \\
(n=300)\end{array}$ & $\begin{array}{l}\text { PBO } \\
(n=78)\end{array}$ & $\begin{array}{l}\mathrm{VDZ}^{\mathrm{a}} \\
(n=334)\end{array}$ & $\begin{array}{l}\text { PBO } \\
(n=18)\end{array}$ & $\begin{array}{l}\mathrm{VDZ}^{\mathrm{a}} \\
(n=112)\end{array}$ \\
\hline Age (years), mean $\pm S D$ & $28.1 \pm 4.7$ & $27.3 \pm 4.6$ & $45.0 \pm 5.5$ & $44.3 \pm 5.9$ & $63.3 \pm 6.0$ & $61.9 \pm 5.8$ \\
\hline Sex (male), $n(\%)$ & $34(64)$ & $179(60)$ & $49(63)$ & $187(56)$ & $9(50)$ & $67(60)$ \\
\hline $\begin{array}{l}\text { Duration of disease (years), } \\
\text { mean } \pm S D\end{array}$ & $4.3 \pm 3.5$ & $4.6 \pm 3.6$ & $8.0 \pm 7.1$ & $8.0 \pm 6.2$ & $11.5 \pm 11.9$ & $9.3 \pm 9.1$ \\
\hline Mayo Clinic score, mean \pm SD & $9.0 \pm 1.7$ & $8.6 \pm 1.7$ & $8.4 \pm 1.7$ & $8.6 \pm 1.8$ & $8.1 \pm 1.6$ & $8.3 \pm 1.6$ \\
\hline Partial Mayo Clinic score, mean $\pm S D$ & $6.4 \pm 1.5$ & $6.1 \pm 1.6$ & $6.0 \pm 1.5$ & $6.0 \pm 1.7$ & $5.6 \pm 1.5$ & $5.7 \pm 1.5$ \\
\hline IBDQ score, mean \pm SD & $124 \pm 33$ & $120 \pm 33$ & $128 \pm 34$ & $122 \pm 32$ & $119 \pm 36$ & $125 \pm 33$ \\
\hline \multicolumn{7}{|l|}{ Disease site } \\
\hline $\begin{array}{l}\text { Rectum and sigmoid colon only, } \\
n(\%)\end{array}$ & $5(9)$ & $40(13)$ & $13(17)$ & $42(13)$ & $4(22)$ & $12(11)$ \\
\hline Left side of colon, $n(\%)$ & $19(36)$ & $91(30)$ & $35(45)$ & $135(40)$ & $5(28)$ & $54(48)$ \\
\hline Proximal to the splenic flexure, $n$ (\%) & $12(23)$ & $43(14)$ & $5(6)$ & $35(10)$ & $1(6)$ & $13(12)$ \\
\hline All of the colon, $n(\%)$ & $17(32)$ & $126(42)$ & $25(32)$ & $122(37)$ & $8(44)$ & $33(29)$ \\
\hline \multicolumn{7}{|l|}{ Concomitant medication for UC } \\
\hline CS only, $n(\%)$ & $21(40)$ & $105(35)$ & $30(38)$ & $128(38)$ & $7(39)$ & $41(37)$ \\
\hline IS only, $n(\%)$ & $5(9)$ & $64(21)$ & $12(15)$ & $56(17)$ & $1(6)$ & $21(19)$ \\
\hline CS and IS, $n(\%)$ & $10(19)$ & $50(17)$ & $13(17)$ & $54(16)$ & $3(17)$ & $19(17)$ \\
\hline No CS or IS, $n(\%)$ & $17(32)$ & $81(27)$ & $23(29)$ & $96(29)$ & $7(39)$ & $31(28)$ \\
\hline $\begin{array}{l}\text { Prednisone equivalent dose }(\mathrm{mg}) \\
\text { median (min, max) }\end{array}$ & $\begin{array}{r}20(5.0 \\
30.0)\end{array}$ & $\begin{array}{r}20(1.0 \\
176.3)\end{array}$ & $\begin{array}{r}20(5.0 \\
40.0)\end{array}$ & $\begin{array}{r}20(0.6 \\
156.3)\end{array}$ & $\begin{array}{l}22.5(5.0 \\
35.0)\end{array}$ & $\begin{array}{r}15(2.5 \\
30.0)\end{array}$ \\
\hline Prior anti-TNF therapy, $n(\%)^{\mathrm{b}}$ & $25(47)$ & $146(49)$ & $38(49)$ & $161(48)$ & $10(56)$ & $51(46)$ \\
\hline Prior anti-TNF failure, $n(\%)^{\mathrm{a}}$ & $21(40)$ & $121(40)$ & $32(41)$ & $140(42)$ & $10(56)$ & $43(38)$ \\
\hline
\end{tabular}

$C S$ corticosteroid, $I B D Q$ inflammatory bowel disease questionnaire, $I S$ immunosuppressant, $P B O$ placebo, $S D$ standard deviation, $T N F$ tumor necrosis factor alpha, $U C$ ulcerative colitis, $V D Z$ vedolizumab

${ }^{a}$ Includes patients from cohort 1 and cohort 2

b Prior anti-TNF exposure was recorded on the interactive voice response system during screening and enrollment. Prior anti-TNF failure was recorded on the case report form at study baseline (week 0). Because of the different data sources, the number of patients with prior anti-TNF exposure does not equal those with prior anti-TNF failure

disease characteristics for the maintenance ITT population were generally consistent with those observed for the induction ITT population, with no important differences between treatment groups.
Approximately $40 \%$ of patients in each age group completed the GEMINI 1 study. Of the patients who enrolled in the GEMINI LTS study, 79 (30\%), 141 (45\%), and 42 (42\%) UC patients aged $<35,35$ to $<55$, and $\geq 55$ years, 
Table 2 CD induction population demographics and baseline disease characteristics

\begin{tabular}{|c|c|c|c|c|c|c|}
\hline \multirow[t]{2}{*}{ Characteristic } & \multicolumn{2}{|c|}{ Age $<35$ years } & \multicolumn{2}{|c|}{ Age 35 to $<55$ years } & \multicolumn{2}{|c|}{ Age $\geq 55$ years } \\
\hline & $\begin{array}{l}\mathrm{PBO} \\
(n=67)\end{array}$ & $\begin{array}{l}\mathrm{VDZ}^{\mathrm{a}} \\
(n=515)\end{array}$ & $\begin{array}{l}\mathrm{PBO} \\
(n=63)\end{array}$ & $\begin{array}{l}\mathrm{VDZ}^{\mathrm{a}} \\
(n=380)\end{array}$ & $\begin{array}{l}\text { PBO } \\
(n=18)\end{array}$ & $\begin{array}{l}\mathrm{VDZ}^{\mathrm{a}} \\
(n=72)\end{array}$ \\
\hline Age (years), mean $\pm S D$ & $27.1 \pm 4.0$ & $26.5 \pm 4.4$ & $43.5 \pm 5.4$ & $43.4 \pm 5.7$ & $64.1 \pm 6.0$ & $61.4 \pm 5.3$ \\
\hline Sex (male), $n(\%)$ & $31(46)$ & $261(51)$ & $31(49)$ & $159(42)$ & $7(39)$ & $31(43)$ \\
\hline $\begin{array}{l}\text { Duration of disease (years), } \\
\text { mean } \pm S D\end{array}$ & $6.1 \pm 4.4$ & $6.6 \pm 4.9$ & $9.4 \pm 7.8$ & $11.5 \pm 8.4$ & $12.1 \pm 13.9$ & $14.9 \pm 12.5$ \\
\hline CDAI score, mean \pm SD & $336 \pm 89$ & $325 \pm 70$ & $318 \pm 67$ & $324 \pm 65$ & $307 \pm 66$ & $308 \pm 63$ \\
\hline IBDQ score, mean \pm SD & $113 \pm 26$ & $121 \pm 30$ & $117 \pm 34$ & $118 \pm 31$ & $112 \pm 36$ & $122 \pm 33$ \\
\hline \multicolumn{7}{|l|}{ Disease site } \\
\hline Ileum only, $n$ (\%) & $6(9)$ & $66(13)$ & $11(17)$ & $73(19)$ & $4(22)$ & $21(29)$ \\
\hline Colon only, $n$ (\%) & $17(25)$ & $136(26)$ & $19(30)$ & $111(29)$ & $7(39)$ & $26(36)$ \\
\hline Ileum and colon, $n$ (\%) & $44(66)$ & $313(61)$ & $33(52)$ & $196(52)$ & $7(39)$ & $25(35)$ \\
\hline \multicolumn{7}{|l|}{ Concomitant medication for CD } \\
\hline CS only, $n(\%)$ & $18(27)$ & $176(34)$ & $18(29)$ & $134(35)$ & $9(50)$ & $26(36)$ \\
\hline IS only, $n(\%)$ & $17(25)$ & $82(16)$ & $7(11)$ & $63(17)$ & $1(6)$ & $11(15)$ \\
\hline CS and IS, $n(\%)$ & $14(21)$ & $98(19)$ & $11(17)$ & $61(16)$ & $1(6)$ & $4(6)$ \\
\hline No CS or IS, $n(\%)$ & $18(27)$ & $159(31)$ & $27(43)$ & $122(32)$ & $7(39)$ & $31(43)$ \\
\hline $\begin{array}{l}\text { Prednisone equivalent dose (mg), } \\
\text { median (min, max) }\end{array}$ & $\begin{array}{l}22.5(5.0 \\
250.0)\end{array}$ & $\begin{array}{r}20(2.5 \\
280.0)\end{array}$ & $\begin{array}{r}20(5.0 \\
35.0)\end{array}$ & $\begin{array}{r}20(2.5 \\
280.0)\end{array}$ & $\begin{array}{c}15(10.0 \\
30.0)\end{array}$ & $\begin{array}{c}17.5(5.0 \\
176.3)\end{array}$ \\
\hline Prior anti-TNF therapy, $n(\%)^{\mathrm{b}}$ & $34(51)$ & $321(62)$ & $30(48)$ & $249(66)$ & $8(44)$ & $47(65)$ \\
\hline Prior anti-TNF failure, $n(\%)^{\mathrm{a}}$ & $35(52)$ & $300(58)$ & $29(46)$ & $231(61)$ & $6(33)$ & $44(61)$ \\
\hline Prior surgery for CD, $n(\%)$ & $24(36)$ & $190(37)$ & $23(37)$ & $181(48)$ & $7(39)$ & $41(57)$ \\
\hline
\end{tabular}

$C D$ Crohn's disease, $C D A I$ Crohn's disease activity index, $C S$ corticosteroid, IBDQ inflammatory bowel disease questionnaire, $I S$ immunosuppressant, $P B O$ placebo, $S D$ standard deviation, $T N F$ tumor necrosis factor alpha, $V D Z$ vedolizumab

a Includes patients from cohort 1 and cohort 2

b Prior anti-TNF exposure was recorded on the interactive voice response system during screening and enrollment. Prior anti-TNF failure was recorded on the case report form at study baseline (week 0). Because of the different data sources, the number of patients with prior anti-TNF exposure does not equal those with prior anti-TNF failure

respectively, were still continuing open-label vedolizumab treatment at 100 weeks post-enrollment (Supplementary Fig. S1a), and $24(9 \%), 27(9 \%)$, and 9 (9\%), respectively, discontinued GEMINI LTS because of an adverse event. In GEMINI 2 study, $48 \%$ of the $\geq 55$-year-old patient population completed the study versus $36 \%$ and $38 \%$ of the $<35$-year-old and 35- to $<55$-year-old patient populations, respectively. Of the $\mathrm{CD}$ patients who enrolled in the GEMINI LTS study, 127 $(35 \%)$ patients $<35$ years old were still continuing treatment at 100 weeks post-enrollment, and 150 (51\%) and 33 (51\%) were continuing at this same time point in the 35 - to $<55$ - and the $\geq 55$-year-old age groups, 
respectively (Supplementary Fig. S1b). Forty-five $(12 \%), 36(12 \%)$, and $5(8 \%)$ patients aged $<35$, 35 to $<55$, and $\geq 55$ years, respectively, discontinued GEMINI LTS because of an adverse event.

\section{Clinical Efficacy in UC}

Vedolizumab induction treatment consistently resulted in numerically greater percentages of UC patients meeting each endpoint at week 6 than placebo (Fig. 2a and Supplementary Fig. S2). At 6 weeks, 44 (51\%), 50 (47\%), and $12(38 \%)$ vedolizumab-treated patients aged $<35,35$ to $<55$, and $\geq 55$ years, respectively, achieved clinical response (Fig. 2a). Further, no differences in treatment benefit were observed between age groups among patients achieving clinical remission or mucosal healing at 6 weeks, with those aged $\geq 55$ years exhibiting the largest 95\% CIs (Supplementary Fig. S2). Of the eight vedolizumab-treated patients aged $\geq 65$ years in the induction ITT group, three achieved a clinical response at week 6 (Supplementary Table S1).

Among vedolizumab-treated patients with response at week 6 who entered the GEMINI 1 maintenance phase, 34 (34\%), 58 (53\%), and 15 $(42 \%)$ who achieved clinical remission at week 52 with continued vedolizumab treatment

(a)

\begin{tabular}{|c|c|c|c|c|c|}
\hline \multirow[b]{2}{*}{ Age, y } & \multicolumn{2}{|c|}{$\begin{array}{l}\text { UC patients in } \\
\text { clinical response } \\
\text { at week } 6, n / N(\%)\end{array}$} & \multicolumn{3}{|c|}{ Difference from PBO, \% (95\% Cl) } \\
\hline & PBO & VDZ & $\frac{\text { Favours }}{\text { PBO }}$ & $\underset{\text { VDZ }}{\stackrel{\text { Favours }}{\longrightarrow}}$ & \\
\hline$<35$ & $11 / 53(20.8)$ & $44 / 86(51.2)$ & & $\longmapsto$ & $30.4(15.2,45.6)$ \\
\hline 35 to $<55$ & $25 / 78(32.1)$ & $50 / 107(46.7)$ & & $\longrightarrow$ & $14.7(0.7,28.7)$ \\
\hline$\geq 55$ & $2 / 18(11.1)$ & $12 / 32(37.5)$ & & $\longrightarrow$ & $26.4(-2.5,52.6)$ \\
\hline
\end{tabular}

(b)

\begin{tabular}{|c|c|c|c|c|c|}
\hline \multirow[b]{2}{*}{ Age, y } & \multicolumn{2}{|c|}{$\begin{array}{l}\text { UC patients in } \\
\text { clinical remission } \\
\text { at week } 52, n / N(\%)\end{array}$} & \multicolumn{3}{|c|}{ Difference from PBO, \% $(95 \% \mathrm{Cl})$} \\
\hline & VDZ/PBO ${ }^{\mathrm{a}}$ & VDZ/VDZ & $\frac{\text { Favours }}{\text { PBO }}$ & $\underset{\text { VDZ }}{\stackrel{\text { Favours }}{\longrightarrow}}$ & \\
\hline$<35$ & $10 / 54(18.5)$ & $34 / 101(33.7)$ & & $\longrightarrow$ & $15.1(1.3,29.0)$ \\
\hline 35 to $<55$ & $6 / 50(12)$ & $58 / 110(52.7)$ & & $\longmapsto$ & $40.7(27.8,53.7)$ \\
\hline$\geq 55$ & $4 / 22(18.2)$ & $15 / 36(41.7)$ & & $\bullet$ & $23.5(-3.2,47.8)$ \\
\hline
\end{tabular}

Fig. 2 Induction and maintenance phase primary endpoints in UC patients by baseline age group. a Clinical response at week 6 in the induction ITT population and $\mathbf{b}$ clinical remission at week 52 in the maintenance ITT population. $C I$ confidence interval, ITT intent-to-treat, $P B O$ placebo, $U C$ ulcerative colitis, $V D Z$

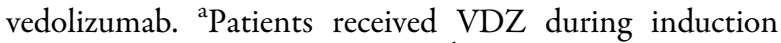
and $\mathrm{PBO}$ during maintenance. ${ }^{\mathrm{b}}$ Patients received VDZ during induction and VDZ every 4 weeks or every 8 weeks during maintenance 
(Fig. 2b). Generally, similar percentages of vedolizumab-treated patients from each age group achieved durable clinical response, durable clinical remission, mucosal healing, and corticosteroid-free remission at week 52, with no noticeable age-related trends (Supplementary Fig. S3). At week 52, clinical remission was achieved in 3 of the 11 patients aged $\geq 65$ years who received vedolizumab every 8 or every 4 weeks during the maintenance phase (Supplementary Table S1).

\section{Clinical Efficacy in CD}

An overall trend favoring vedolizumab treatment was observed in the induction and maintenance phases of the GEMINI 2 study. At week 6 , percentages of vedolizumab-treated patients who achieved clinical remission in the $<35$-year-old (14\%) and the 35- to $<55$-year-old $(16 \%)$ age groups were similar and higher than in the $\geq 55$-year-old age group (8\%) (Fig. 3a). The percentage of vedolizumab-treated patients with enhanced clinical response increased with increasing age group $(28 \%, 33 \%$, and $46 \%$, respectively) (Fig. 3b). Of the two vedolizumab-treated patients $\geq 65$ years old in the induction ITT group, one achieved enhanced clinical response and clinical remission at week 6 (Supplementary Table S2). At 6 weeks, the mean change from baseline serum CRP levels did not differ with vedolizumab treatment across the age groups (Supplementary Table S3).

After re-randomization of patients with clinical response at week 6 , similar percentages of vedolizumab-treated patients in all age subgroups achieved clinical remission (Fig. 3c), enhanced clinical response (Supplementary Fig. S4), and corticosteroid-free remission (Supplementary Fig. S4) at week 52. Vedolizumab maintenance treatment resulted in $20 \%, 19 \%$, and $9 \%$ of patients aged $<35,35$ to $<55$, and $\geq 55$ years, respectively, achieving durable clinical remission (Supplementary Fig. S4). Two of the five patients $\geq 65$ years old achieved clinical remission in the group that received vedolizumab every 8 or every 4 weeks (Supplementary Table S2).

\section{Safety in UC and CD}

The safety profile of vedolizumab in the total combined GEMINI 1 and GEMINI 2 population was similar across all age groups investigated. The most common adverse events reported were nasopharyngitis, headache, arthralgia, and nausea, each occurring with an incidence of $\geq 10$ patients per $100 \mathrm{PY}$ in the combined vedolizumab-exposed patient population in each age group (Table 3). Of these, in patients $\geq 55$ years old, the incidence of nasopharyngitis, nausea, and upper respiratory tract infection, as well as pyrexia, appeared higher in vedolizumab-treated patients than in the placebo group (Table 3 ). Patients $\geq 65$ years old reported a generally similar adverse event profile to patients $<65$ years old (Supplementary Table S4).

The rates of adverse events were generally similar across all age groups. Rates of any adverse events per $100 \mathrm{PY}$ were 337.8, 364.6, and 343.8 in vedolizumab-treated patients aged $<35,35$ to $<55$, and $\geq 55$ years, respectively, versus $482.6,323.6$, and 394.3 in patients who received placebo (Table 3). Infusion-related reactions were uncommon and occurred at similar rates throughout the age and treatment groups (Table 3). Psoriasis-an adverse event observed with anti-TNF therapy [26]—in particular, was reported in $<2$ patients per $100 \mathrm{PY}$ in any treatment group, irrespective of patient age. Memory impairment, when captured as an adverse event verbatim, was reported for 7 (1.4 per $100 \mathrm{PY}), 8$ (1.8 per $100 \mathrm{PY})$, and 4 (3.6 per $100 \mathrm{PY})$ vedolizumab-treated patients aged $<35,35$ to $<55$, and $\geq 55$ years, respectively, versus 0,1 (1.2 per $100 \mathrm{PY}$ ), and 1 (4.3 per $100 \mathrm{PY}$ ) patients who received placebo.

In general, rates of infections were similar across all age groups (Table 3). No patient $\geq 55$ years of age in either the vedolizumab- or placebo-exposed group had a clostridia infection versus $<0.7$ per $100 \mathrm{PY}$ in any combined vedolizumab-treated population aged $<35$ or 35 to $<55$ years. The incidence of pneumonia was $\leq 1.4$ per $100 \mathrm{PY}$ in vedolizumab-treated patients in any age group. 
(a)

\begin{tabular}{|c|c|c|c|c|c|}
\hline \multirow[b]{2}{*}{ Age, y } & \multicolumn{2}{|c|}{$\begin{array}{l}\text { CD patients in } \\
\text { clinical remission } \\
\text { at week } 6, n / N(\%)\end{array}$} & \multicolumn{3}{|c|}{ Difference from PBO, \% (95\% Cl) } \\
\hline & PBO & VDZ & PBO & $\underset{\mathrm{VDZ}}{\mathrm{P}}$ & \\
\hline$<35$ & $5 / 67(7.5)$ & 16/111 (14.4) & $\boldsymbol{r}$ & -1 & $7.0(-8.1,22.0)$ \\
\hline 35 to $<55$ & $3 / 63(4.8)$ & 15/96 (15.6) & & $\leadsto$ & $10.9(-5.0,26.5)$ \\
\hline$\geq 55$ & $2 / 18(11.1)$ & $1 / 13(7.7)$ & & $\longrightarrow$ & $-3.4(-37.5,31.8)$ \\
\hline
\end{tabular}

(b)

\begin{tabular}{|c|c|c|c|c|c|}
\hline \multirow[b]{2}{*}{ Age, y } & \multicolumn{2}{|c|}{$\begin{array}{c}\text { CD patients with } \\
\text { enhanced clinical response } \\
\text { at week } 6, n / N(\%)\end{array}$} & \multicolumn{3}{|c|}{ Difference from PBO, \% $(95 \% \mathrm{Cl})$} \\
\hline & PBO & VDZ & $\frac{\text { favours }}{\text { PBO }}$ & $\underset{\text { VDZ }}{\text { Favours }}$ & \\
\hline$<35$ & $21 / 67(31.3)$ & 31/111 (27.9) & 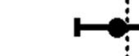 & $\rightarrow$ & $-3.4(-17.3,10.5)$ \\
\hline 35 to $<55$ & $14 / 63(22.2)$ & 32/96 (33.3) & & $\rightarrow$ & $11.1(-2.8,25.1)$ \\
\hline$\geq 55$ & $3 / 18(16.7)$ & $6 / 13(46.2)$ & & $\longrightarrow$ & $29.5(-6.4,60.6)$ \\
\hline
\end{tabular}

(c)

\begin{tabular}{|c|c|c|c|c|c|}
\hline \multirow[b]{2}{*}{ Age, y } & \multicolumn{2}{|c|}{$\begin{array}{l}\text { CD patients in } \\
\text { clinical remission } \\
\text { at week } 52, n / N(\%)\end{array}$} & \multicolumn{3}{|c|}{ Difference from PBO, \% (95\% Cl) } \\
\hline & VDZ/PBO ${ }^{a}$ & VDZ/VDZ ${ }^{b}$ & $\frac{\text { Favours }}{\mathrm{PBO}}$ & $\frac{\text { Favours }}{\mathrm{VDZ}}$ & \\
\hline$<35$ & 15/73 (20.5) & $65 / 173(37.6)$ & & $\mapsto-1$ & $17.0(5.3,28.8)$ \\
\hline 35 to $<55$ & $13 / 65(20.0)$ & $42 / 112(37.5)$ & & $\longmapsto$ & $17.5(4.3,30.7)$ \\
\hline$\geq 55$ & $5 / 15(33.3)$ & 9/23 (39.1) & $\vdash$ & $\longrightarrow$ & $5.8(-26.5,37.6)$ \\
\hline
\end{tabular}

There were no reports of progressive multifocal leukoencephalopathy.

No increase in the occurrence of diabetes was apparent with age or with vedolizumab compared with placebo. Type 2 diabetes mellitus was reported with vedolizumab treatment in one patient $<35$ years old $(0.2$ per $100 \mathrm{PY}$ ) and one patient $\geq 55$ years old ( 0.9 per 
4Fig. 3 Induction and maintenance phase primary endpoints in CD patients by baseline age group. a Clinical remission at week 6 in the induction ITT population, $\mathbf{b}$ enhanced clinical response at week 6 in the induction ITT population, and c clinical remission at week 52 in the maintenance ITT population. $C D$ Crohn's disease, $C I$ confidence interval, ITT intent-to-treat, $P B O$ placebo, $V D Z$ vedolizumab. ${ }^{\text {P }}$ Patients received $V D Z$ during induction and $\mathrm{PBO}$ during maintenance. ${ }^{\mathrm{b}}$ Patients received VDZ during induction and VDZ every 4 weeks or every 8 weeks during maintenance

$100 \mathrm{PY}$ ). Blood glucose increased in $<0.7$ patients per $100 \mathrm{PY}$ in all vedolizumabexposed age groups and $<1.2$ in all placebo-exposed age groups analyzed. In addition, no meaningful differences were observed in the percentage of patients with adverse hematological events, in general, between vedolizumab and placebo treatment in any age group (Supplementary Table S5).

Similarly, the occurrence of serious adverse events, including serious infections, was generally consistent across all age groups (Table 3). However, the incidence of serious adverse events in vedolizumab-treated patients was lowest in patients aged $\geq 55$ years, with serious events reported in 17 patients aged $\geq 55$ years (15.9 per $100 \mathrm{PY})$ versus 149 (32.1 per PY) and 110 (27.5 per PY) patients aged $<35$ and 35 to $<55$ years, respectively (Table 3 ). Compared with younger vedolizumab-treated patients, those receiving vedolizumab in the $\geq 55$-year-old age group had the lowest incidence of adverse events leading to hospitalizations (Table 3). The most common adverse events that led to hospitalization in vedolizumab-treated patients aged $<35,35$ to $<55$, and $\geq 55$ years were exacerbation of UC (3\%, $4 \%$, and $1 \%$ of patients, respectively) and CD (10\%, $4 \%$, and $3 \%$ of patients, respectively). No other individual adverse event led to hospitalization in $>2 \%$ of vedolizumab-treated patients in any age group.

Malignancies were reported in two vedolizumab-treated patients aged $<35$ years (0.4 per $100 \mathrm{PY})$, two vedolizumab-treated patients aged 35 to $<55$ years ( 0.5 per $100 \mathrm{PY}$ ), and one patient who received placebo aged $\geq 55$ years ( 4.3 per $100 \mathrm{PY}$ ) (Table 3 ). There were two additional malignancies reported in the maintenance ITT placebo population (Table 4), a group not represented in the safety population in Table 3. Overall, there was no apparent relationship to age in the number or type of malignancy reported. All patients with malignancy had risk factors that may have contributed to the etiology, including prior therapy with immunosuppressives, smoking history, and/or history of a prior cancer or related medical condition. Of the two patients aged $\geq 55$ years, one had received only placebo before being diagnosed with squamous cell carcinoma of the nose, which was resolved following excision. The second patient, a 73-year-old male with colon cancer, had a 14-year history of UC limited to the rectum and sigmoid colon. Both patients had previously received azathioprine/ mercaptopurine and corticosteroids (rectal and systemic).

Six deaths occurred during the studies: three vedolizumab-treated $\mathrm{CD}$ patients aged $<35$ years, one vedolizumab-treated $\mathrm{CD}$ patient aged 46 years, and two patients $\geq 55$ years old, one of whom, a 75 -year-old CD patient, had received only placebo (Supplementary Table S6). A 66-year-old male UC patient died of reported acute cardiac death 14 days after his first induction dose of open-label vedolizumab. His medical record included a 2-year history of chronic ischemic heart disease, and the postmortem examination showed diffuse multifocal cardiosclerosis. Detailed accounts of all deaths have been described previously $[22,23]$.

\section{DISCUSSION}

The post hoc analyses we report descriptively show that the efficacy of vedolizumab induction and maintenance therapy for UC and $C D$ is generally similar between patients aged $<35,35$ to $<55$, and $\geq 55$ years, and no age-related differences were observed in the adverse event profile. As would be expected, disease duration was longer in the older GEMINI patients; however, an increase in 


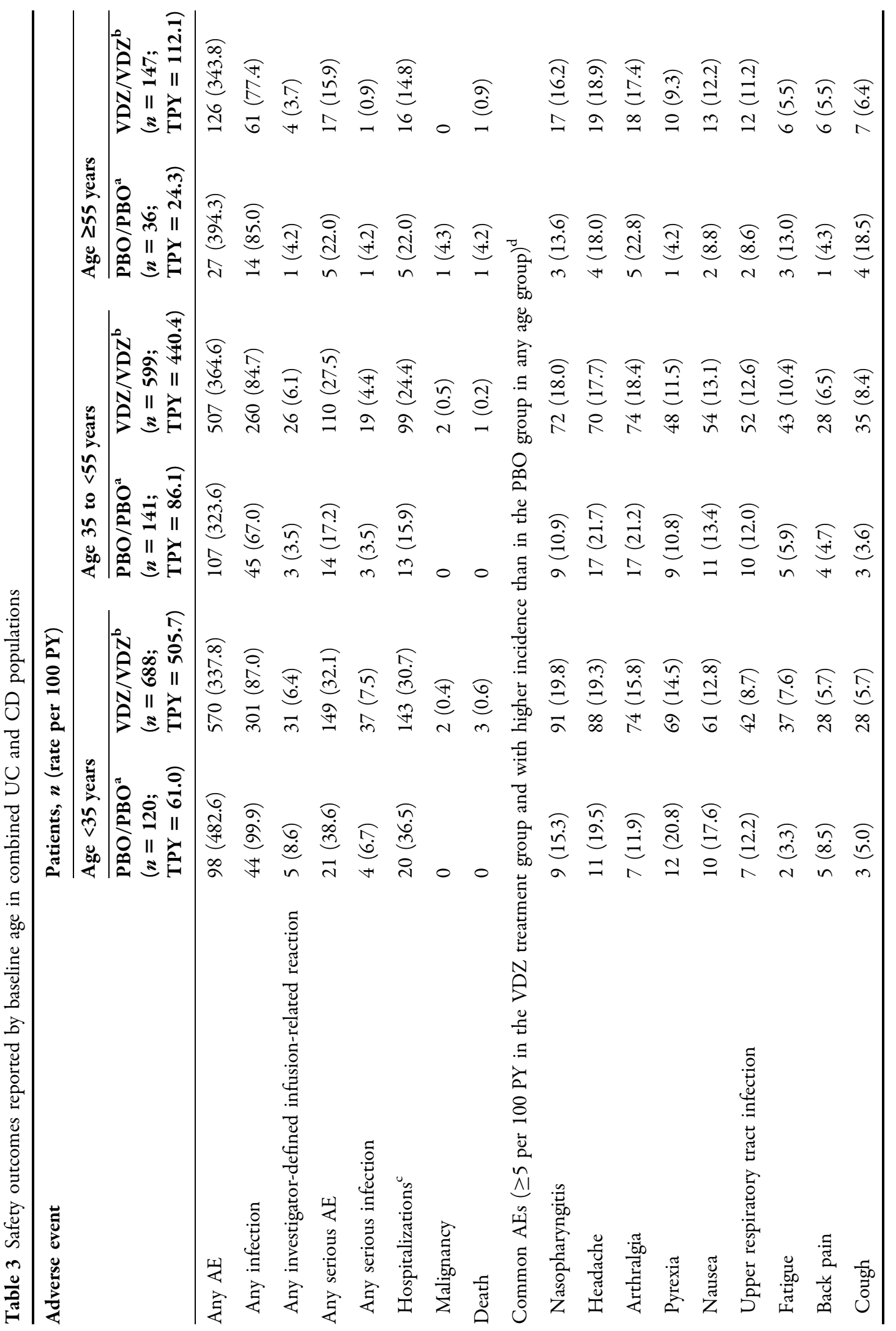




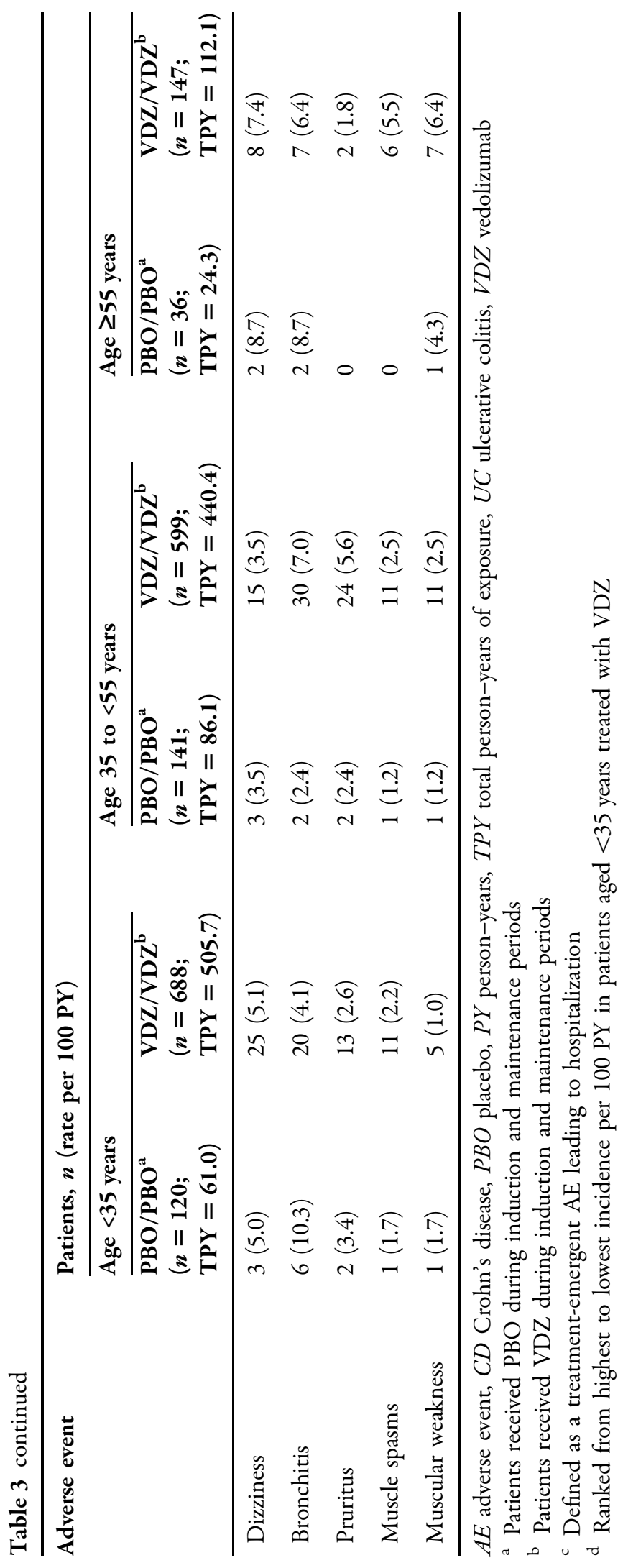


Table 4 Malignancies reported in patients with UC and CD

\begin{tabular}{|c|c|c|c|c|c|c|}
\hline Age & $\begin{array}{l}\text { No. of } \\
\text { events }\end{array}$ & Age/sex & Indication & Neoplasm & $\begin{array}{l}\text { Treatment group } \\
\text { (induction/maintenance) }\end{array}$ & $\begin{array}{l}\text { No. of VDZ } \\
\text { doses }\end{array}$ \\
\hline \multirow[t]{2}{*}{$<35$ years } & 2 & $20.7 / \mathrm{F}$ & $\mathrm{CD}$ & $\begin{array}{l}\text { Carcinoid tumor of } \\
\text { the appendix }\end{array}$ & VDZ/VDZ (Q4 W) & 14 \\
\hline & & $32.7 / \mathrm{M}$ & UC & Colon cancer & VDZ/VDZ (Q8 W) & 7 \\
\hline \multirow[t]{3}{*}{$\begin{array}{l}35 \text { to } \\
<55 \text { years }\end{array}$} & 3 & $40.5 / \mathrm{M}$ & UC & $\begin{array}{l}\text { Transitional cell } \\
\text { carcinoma }\end{array}$ & $\mathrm{VDZ} / \mathrm{PBO}$ & $2^{a}$ \\
\hline & & $45.2 / \mathrm{F}$ & $\mathrm{CD}$ & Breast cancer & $\begin{array}{l}\text { Cohort } 2 \text { VDZ (induction } \\
\text { only) }\end{array}$ & 2 \\
\hline & & $52.1 / \mathrm{F}$ & $\mathrm{CD}$ & $\begin{array}{l}\text { Squamous cell } \\
\text { carcinoma (skin) }\end{array}$ & VDZ/VDZ (Q4 W) & 11 \\
\hline \multirow[t]{2}{*}{$\geq 55$ years } & 2 & $70.7 / \mathrm{F}$ & UC & $\begin{array}{l}\text { Squamous cell } \\
\text { carcinoma }\end{array}$ & $\mathrm{PBO} / \mathrm{PBO}$ & 0 \\
\hline & & $73.7 / \mathrm{M}$ & UC & Colon cancer & $\mathrm{VDZ} / \mathrm{PBO}$ & $2^{a}$ \\
\hline
\end{tabular}

$C D$ Crohn's disease, ITT intent-to-treat, $P B O$ placebo, $Q 4 W$ every 4 weeks, $Q 8 W$ every 8 weeks, $U C$ ulcerative colitis, $V D Z$ vedolizumab

${ }^{\text {a }}$ Received 2 doses of VDZ during induction

disease severity was not observed with longer disease duration, as evidenced by baseline disease activity scores. Thus, the consistent efficacy and safety observed with increasing age were not confounded by variations in disease severity. These data are promising and especially relevant because of the rapidly rising global population of patients over the age of 60 [27]. However, both studies enrolled small numbers of patients $\geq 65$ years of age and therefore further studies of the safety and efficacy of vedolizumab in the elderly population are needed.

There are currently no formal guidelines for the treatment of IBD in elderly populations, leaving substantial gaps in the treatment paradigm [5]. Elderly patients are underrepresented in clinical trials, and the sparse data that are available for this population indicate that managing elderly patients with IBD is complex and disparate from IBD management in younger patients. Factors that contribute to the complexity include the potential for a smaller response to treatment, altered clinical course of IBD, and increased risk of malignancies and infections [5].
Clinical trials often exclude the elderly population either explicitly or implicitly through exclusion criteria and intensity of study-related visits. As a result, older patients are not well represented in phase 3 clinical trials [3]. Indeed, the median patient age in most IBD studies is generally around the thirties [3]. Similarly, neither GEMINI 1 nor GEMINI 2 was designed to investigate these subgroups specifically. Thus, these post hoc analyses are limited by small sample size and are not adequately powered to detect statistically significant differences. However, the efficacy of vedolizumab in UC and CD patients relative to placebo was overall consistent across all endpoints, irrespective of the patient's age group. Further, experience with anti-TNF therapy has shown an inverse relationship between disease duration and clinical efficacy [28], suggesting that efficacy may decline as patients age. In the vedolizumab GEMINI 1 and GEMINI 2 studies, patients aged $\geq 55$ years had longer disease duration than their younger counterparts but experienced similar clinical benefits, although some variability between subgroups was observed. For example, no 
difference was observed between vedolizumab and placebo treatment in the percentages of CD patients with corticosteroid-free remission at week 52 among those aged $\geq 55$ years. However, this observation in CD could be attributed to the small patient population with corticosteroid use at baseline in this age group (seven patients in each treatment group). Indeed, wide 95\% CIs were associated with the percentage-point differences from placebo in the majority of endpoints for patients aged $\geq 55$ years, reflecting variability in the data likely due to the small number of patients in these post hoc subgroup analyses. Corticosteroid sparing is particularly important for older IBD patients since long-term, high-frequency or high-dose corticosteroid use in these patients often exacerbates conditions such as congestive heart failure, diabetes, hypertension, osteoporosis, psychosis, depression, electrolyte abnormalities, cataracts, glaucoma, or infections [14, 18]. Yet chronic corticosteroid use coupled with infrequent attempts to taper is a common practice in elderly IBD patients aged $\geq 65$ years $[14,29]$. Importantly, the absolute percentages of patients achieving corticosteroid-free remission with vedolizumab maintenance treatment at week 52 were similar for all UC patients (33-42\%) and CD patients (27-33\%) regardless of age.

A recent integrated study of the vedolizumab clinical trials, including GEMINI 1 and GEMINI 2 , found younger age was a risk factor for serious infection with vedolizumab exposure in a combined population of 2884 patients with UC or CD (hazard ratio 0.98; 95\% CI 0.97-0.99; $P<0.001)$ but did not report any other safety-age relationships [24]. Indeed, we observed 10 fewer patients with infection per $100 \mathrm{PY}$ of vedolizumab in the $\geq 55$-year-old age group than in the $<35$-year-old age group. Further, we found no evidence of a change in occurrence of malignancy, diabetes, other adverse hematological events, or infusion-related reactions with vedolizumab treatment in patients with advancing age from $<35$ to $\geq 55$ years. No major age-related trends in the occurrence of the most common adverse events reported were observed. While cognitive function was not evaluated directly in GEMINI
1 and GEMINI 2, the occurrence of an adverse event of memory impairment with vedolizumab was low [4 patients $(3 \%$; 3.6 per $100 \mathrm{PY}$ ) aged $\geq 55$ years] and similar to that observed with placebo [1 patient (3\%; 4.3 per 100 PY) aged $\geq 55$ years] in all age groups investigated. One malignancy was reported in a patient $\geq 55$ years of age exposed to vedolizumab induction; however, patient risk factors (i.e., a history of tobacco use, a long duration of UC, and prior therapy with immunosuppressives and corticosteroids) may have contributed to the etiology. Recently, a retrospective study of 14,733 patients with moderately to severely active IBD reported an incidence of 0.7 per $100 \mathrm{PY}$ for primary solid tumors [30]. In comparison, the incidence of cancer was $\leq 0.5$ per $100 \mathrm{PY}$ in our combined vedolizumab-exposed population. Notably, a 1-year controlled study may not be long enough to detect malignancies and other adverse events that usually take longer to develop. Continued monitoring through epidemiological studies and patient registries will help evaluate the long-term safety profile of vedolizumab. Of note, percentages of UC and CD patients $\geq 55$ years of age who completed the 52-week GEMINI 1 and GEMINI 2 studies and of those who were continuing in the open-label GEMINI LTS trial at 100 weeks post-enrollment were similar to those of patients $<55$ years of age. In contrast, anti-TNF therapies have been linked to premature discontinuation in older IBD patients more often than younger patients $[10,31]$. In one retrospective single-center study, $53 \%$ of patients aged $>60$ years were continuing anti-TNF therapy at 12 months after initiation versus $90 \%$ of younger users [10]. The increased susceptibility of older patients to infectious complications and adverse events, in general, has been proposed as an underlying reason for premature treatment cessation in this population [10, 31].

Overall, the current analysis highlighted important trends in the data favoring vedolizumab over placebo for most efficacy endpoints irrespective of age. Safety profiles were found to be similar between vedolizumab and placebo in all age groups. However, given 
the limitations of this study, further investigation of the safety and efficacy of vedolizumab in the elderly population is needed. As mentioned earlier, the current results are based on a post hoc subgroup analysis of data from the GEMINI 1 and GEMINI 2 studies, both of which were limited in size (particularly for patients $\geq 65$ years of age) and duration; and therefore they were not powered for efficacy or safety analyses [22, 23]. Further, as a result of the multiplicity of adverse events and the spontaneous nature of adverse event reporting, descriptive statistics were used to compare the incidences of adverse events between patient groups in the original pivotal studies and the current subgroup analyses. Finally, comorbidities, especially as patients age, may confound study results; however, monitoring of comorbidities was not prespecified in the trial designs, making stratification of patients based on comorbid conditions challenging.

\section{CONCLUSIONS}

Because of changing demographics, the safe and effective treatment of older IBD patients will be an ongoing challenge. The results of these post hoc analyses suggest that vedolizumab treatment was efficacious and well tolerated in IBD patients across all ages. Based on these results, and within the limitation of these analyses, vedolizumab may offer an attractive alternative to the currently available treatment options. In the absence of prospective interventional studies investigating the effects of vedolizumab in elderly patients, the emergence of real-world data will add to the findings of these post hoc analyses and provide further support for whether vedolizumab is a suitable treatment option for elderly IBD patients.

\section{ACKNOWLEDGEMENTS}

Sponsorship, article processing charges, and the open access charge for this study were funded by Millennium Pharmaceuticals, Inc. (d/b/a Takeda Pharmaceuticals International Co.).
Medical writing assistance was provided by inVentiv Medical Communications and supported by Takeda Pharmaceuticals International Co. Publication management support was provided by Caterina Hatzifoti Ph.D. of Takeda Pharmaceuticals International. All named authors meet the International Committee of Medical Journal Editors (ICMJE) criteria for authorship for this manuscript, had full access to all of the data in this study, and take complete responsibility for the integrity of the data and accuracy of the data analysis.

Disclosures. Vijay Yajnik has served as a consultant for AbbVie, Biogen, Janssen, NPS Pharma, Takeda, and UCB. Nabeel Khan has received financial support for research from Takeda. Marla Dubinsky has served as a consultant for Abbott, Bristol-Myers Squibb Co, Janssen-Cilag, Pfizer Inc, Prometheus Laboratories Inc, Takeda, and UCB; and received financial support for research from Janssen-Cilag. Jeffrey Axler served as a consultant for AbbVie, Janssen Pharmaceutical, and Takeda. Alexandra James is an employee of Takeda Development Centre Europe Ltd, London, UK. Brihad Abhyankar is an employee of Takeda Development Centre Europe Ltd, London, UK. Karen Lasch is an employee of Takeda Pharmaceuticals International Inc, Deerfield, IL, USA.

Compliance with Ethics Guidelines. The study protocol and the informed consent documentation were reviewed and approved by the institutional review board(s) or independent ethics committee(s) at each investigational center. The study was conducted in compliance with the protocol, good clinical practice, and the applicable regulatory requirements (including International Conference on Harmonisation guidelines), and in accordance with ethical principles founded in the Declaration of Helsinki of 1964, as revised in 2013. Written informed consent for being included in the study was obtained from all patients at the time they entered the screening process.

Data Availability. All data generated or analyzed during this study are included in this 
published article/as supplementary information files.

Open Access. This article is distributed under the terms of the Creative Commons Attribution-NonCommercial 4.0 International License (http://creativecommons.org/licenses/ by-nc/4.0/), which permits any noncommercial use, distribution, and reproduction in any medium, provided you give appropriate credit to the original author(s) and the source, provide a link to the Creative Commons license, and indicate if changes were made.

\section{REFERENCES}

1. Baumgart DC, Sandborn WJ. Crohn's disease. Lancet. 2012;380(9853):1590-605.

2. Molodecky NA, Soon IS, Rabi DM, et al. Increasing incidence and prevalence of the inflammatory bowel diseases with time, based on systematic review. Gastroenterology. 2012;142(1):46-54.

3. Katz S, Pardi DS. Inflammatory bowel disease of the elderly: frequently asked questions (FAQs). Am J Gastroenterol. 2011;106(11):1889-97.

4. Ruel J, Ruane D, Mehandru S, Gower-Rousseau C, Colombel JF. IBD across the age spectrum: is it the same disease? Nat Rev Gastroenterol Hepatol. 2014;11(2):88-98.

5. Ha CY, Katz S. Clinical implications of ageing for the management of IBD. Nat Rev Gastroenterol Hepatol. 2014;11(2):128-38.

6. Brassard P, Bitton A, Suissa A, Sinyavskaya L, Patenaude V, Suissa S. Oral corticosteroids and the risk of serious infections in patients with elderly-onset inflammatory bowel diseases. Am J Gastroenterol. 2014;109(11):1795-802.

7. Gisbert JP, Chaparro M. Systematic review with meta-analysis: inflammatory bowel disease in the elderly. Aliment Pharmacol Ther. 2014;39(5):459-77.

8. Cottone M, Kohn A, Daperno M, et al. Advanced age is an independent risk factor for severe infections and mortality in patients given anti-tumor necrosis factor therapy for inflammatory bowel disease. Clin Gastroenterol Hepatol. 2011;9(1):30-5.
9. Lobaton T, Ferrante M, Rutgeerts P, Ballet V, Van Assche G, Vermeire S. Efficacy and safety of anti-TNF therapy in elderly patients with inflammatory bowel disease. Aliment Pharmacol Ther. 2015;42(4):441-51.

10. Desai A, Zator ZA, de Silva $P$, et al. Older age is associated with higher rate of discontinuation of anti-TNF therapy in patients with inflammatory bowel disease. Inflamm Bowel Dis. 2013;19(2):309-15.

11. Toruner M, Loftus EV Jr, Harmsen WS, et al. Risk factors for opportunistic infections in patients with inflammatory bowel disease. Gastroenterology. 2008;134(4):929-36.

12. Beaugerie L, Brousse $\mathrm{N}$, Bouvier AM, et al. Lymphoproliferative disorders in patients receiving thiopurines for inflammatory bowel disease: a prospective observational cohort study. Lancet. 2009;374(9701):1617-25.

13. Ananthakrishnan AN, Cagan A, Cai T, et al. Diabetes and the risk of infections with immunomodulator therapy in inflammatory bowel diseases. Aliment Pharmacol Ther. 2015;41(11):1141-8.

14. Juneja M, Baidoo L, Schwartz MB, et al. Geriatric inflammatory bowel disease: phenotypic presentation, treatment patterns, nutritional status, outcomes, and comorbidity. Dig Dis Sci. 2012;57(9):2408-15.

15. Stallmach A, Hagel S, Gharbi A, et al. Medical and surgical therapy of inflammatory bowel disease in the elderly-prospects and complications. J Crohns Colitis. 2011;5(3):177-88.

16. Khan N, Abbas AM, Lichtenstein GR, Loftus EV Jr, Bazzano LA. Risk of lymphoma in patients with ulcerative colitis treated with thiopurines: a nationwide retrospective cohort study. Gastroenterology. 2013;145(5):1007-15.

17. Peyrin-Biroulet L, Khosrotehrani K, Carrat F, et al. Increased risk for nonmelanoma skin cancers in patients who receive thiopurines for inflammatory bowel disease. Gastroenterology. 2011;141(5): 1621-8.

18. Sales-Campos H, Basso PJ, Alves VB, et al. Classical and recent advances in the treatment of inflammatory bowel diseases. Braz J Med Biol Res. 2015;48(2):96-107.

19. Fardet L, Feve B. Systemic glucocorticoid therapy: a review of its metabolic and cardiovascular adverse events. Drugs. 2014;74(15):1731-45.

20. Soler D, Chapman T, Yang LL, Wyant T, Egan R, Fedyk ER. The binding specificity and selective 
antagonism of vedolizumab, an anti- $\alpha_{4} \beta_{7}$ integrin therapeutic antibody in development for inflammatory bowel diseases. J Pharmacol Exp Ther. 2009;330(3):864-75.

21. Nasa P, Juneja D, Singh O. Severe sepsis and septic shock in the elderly: an overview. World J Crit Care Med. 2012;1(1):23-30.

22. Feagan BG, Rutgeerts $P$, Sands BE, et al. Vedolizumab as induction and maintenance therapy for ulcerative colitis. $\mathrm{N}$ Engl J Med. 2013;369(8):699-710.

23. Parikh A, Fox I, Leach $\mathrm{T}$, et al. Long-term clinical experience with vedolizumab in patients with inflammatory bowel disease. Inflamm Bowel Dis. 2013;19(8):1691-9.

24. Colombel JF, Sands BE, Rutgeerts P, et al. The safety of vedolizumab for ulcerative colitis and Crohn's disease. Gut. 2016. doi:10.1136/gutjnl-2015311079 .

25. Brown EG, Wood L, Wood S. The medical dictionary for regulatory activities (MedDRA). Drug Saf. 1999;20(2):109-17.

26. Joyau C, Veyrac G, Dixneuf $V$, Jolliet $P$. Anti-tumour necrosis factor alpha therapy and increased risk of de novo psoriasis: is it really a paradoxical side effect? Clin Exp Rheumatol. 2012;30(5):700-6.

27. Scult M, Haime V, Jacquart J, et al. A healthy aging program for older adults: effects on self-efficacy and morale. Adv Mind Body Med. 2015;29(1):26-33.

28. Schreiber S, Colombel JF, Bloomfield R, et al. Increased response and remission rates in short-duration Crohn's disease with subcutaneous certolizumab pegol: an analysis of PRECiSE 2 randomized maintenance trial data. Am J Gastroenterol. 2010;105(7):1574-82.

29. Parian A, Ha CY. Older age and steroid use are associated with increasing polypharmacy and potential medication interactions among patients with inflammatory bowel disease. Inflamm Bowel Dis. 2015;21(6):1392-400.

30. McAuliffe M, Lanes S, Leach T, et al. Occurrence of adverse events among patients with inflammatory bowel disease in the HealthCore Integrated Research Database. Curr Med Res Opin. 2015;31(9):1655-64.

31. Nimmons D, Limdi JK. Elderly patients and inflammatory bowel disease. World J Gastrointest Pharmacol Ther. 2016;7(1):51-65. 